\title{
Effect of Parathyroidectomy on Quality of Life Among Patients Undergoing Dialysis
}

\author{
Lin Wang', Ming-Hui Xin', Yan Ma', Yu Wang', Meng-Yuan Hu', Qiang-Qiang Liu', Jin-Bor Chen ${ }^{2,3}$ \\ 'Department of Nephrology, Dalian University Affiliated Xinhua Hospital, Dalian, People's Republic of China; ${ }^{2}$ Division of Nephrology, Department of \\ Internal Medicine, Kaohsiung Chang Gung Memorial Hospital, Kaohsiung City, Taiwan, Republic of China; ${ }^{3}$ School of Medicine, College of Medicine, \\ Chang Gung University, Taoyuan, Taiwan, Republic of China \\ Correspondence: Jin-Bor Chen, Division of Nephrology, Department of Internal Medicine, Kaohsiung Chang Gung Memorial Hospital, 123 Ta Pei Road, Niao \\ Song District, Kaohsiung City, Taiwan, Republic of China, Tel +886-7-73 I7I23, ext 8306, Fax +886-7-7322402, Email chenjb1019@gmail.com
}

\begin{abstract}
Purpose: There is a limited evidence of durable effect of parathyroidectomy (PTX) on the quality of life (QoL) in dialysis populations. We aimed to investigate this concern by comparing the QoL scores in the pre- and post-PTX periods in a cohort of dialysis patients.

Patients and Methods: A total of 212 dialysis patients were enrolled in a hospital-facilitated dialysis center in China between July 1, 2016 and June 30, 2021. The mean age was 46.4 years; the male:female ratio was 96:116; hemodialysis 191, peritoneal dialysis 21. Informative data relating to demographics and dialysis were recorded for comparison. QoL was measured using the Chinese version of the Kidney Disease Quality of Life-36 (KDQOL-36 ${ }^{\mathrm{TM}}$ ) and compared subscale scores between the pre-and post-PTX period. Appropriate statistical methods and Pearson's correlation test were used for statistical analysis.

Results: Nutritional markers, including hemoglobin and albumin, significantly increased post-PTX than pre-PTX. KDQOL-36 domain scale scores, including Symptoms and Problems of Kidney Disease, Burden of Kidney Disease, Effects of Kidney Disease (EKD), Physical Component Summary (PCS) score, and Mental Component Summary score, significantly increased post-PTX than pre-PTX. All patients were further stratified into three groups based on the PTX duration $-0-2$ years, $>2-<5$ years, and $\geq 5$ years - and all KDQOL-36 domain scale scores increased in individual PTX durations. The PTX duration showed a significant negative correlation between PCS subscale scores and a positive correlation between EKD subscale scores.
\end{abstract}

Conclusion: PTX could improve QoL in dialysis patients with medically refractory secondary hyperparathyroidism. The durable effects should be studied using a larger sample.

Keywords: dialysis, Kidney Disease Quality of Life-36, parathyroidectomy

\section{Introduction}

Elevated circulating parathyroid hormone (PTH) concentrations commonly appear in chronic kidney disease (CKD), and secondary hyperparathyroidism (HPT) is used to describe this complication. Insights into the complex pathogenesis of increased PTH in CKD have improved in the past decades. ${ }^{1,2}$ Given this achievement, it is well recognized that PTH can elicit untoward biological effects, such as mineral bone disease, soft tissue and vascular calcification, cardiac and skeletal myopathy, unfavorable anemia, cognitive impairment, and sleep disturbance. ${ }^{1,3-8}$ Clinical observational studies have also demonstrated increased mortality risk in CKD with elevated PTH concentrations. ${ }^{9-12}$

Recently, quality of life (QoL) has been emphasized in CKD, especially in dialysis patient care. Symptoms in CKD itself and CKD treatment influence QoL in the CKD population. ${ }^{13}$ Clinical manifestations of secondary HPT confer QoL to somatic complaints or psychological disturbances. The Kidney Disease Improving Global Outcomes (KDIGO) guidelines recommend that parathyroidectomy (PTX) is indicated in patients with high PTH concentrations $(>800 \mathrm{pg} / \mathrm{mL})$ and patients refractory to medical treatment. ${ }^{1}$ It is reasonable to assume that PTX relieves and/or alleviates clinical complaints in CKD patients with secondary HPT. Thus, PTX has a positive effect on QoL in patients with CKD undergoing dialysis. 
Importantly, several clinical observational studies have demonstrated the role of the intrinsic value of PTX in QoL improvement in end-stage renal disease-related HPT., ${ }^{414-18}$

Patient-reported outcome measurements are often used to assess and monitor the QoL of patients with disease. In dialysis patients, the Kidney Disease Quality of Life 36-item short form survey (KDQOL-36) is one of the most widely used tools to measure QoL in patients with end-stage renal disease. ${ }^{19}$ The measurements include both generic and disease-specific components for QoL assessment. It offers an instrument to detect treatment effects and is responsive to changes in specific conditions for end-stage renal disease.

Herein, we investigated a longitudinal PTX effect on QoL in a cohort of dialysis patients. Our study aimed to compare psychometric and somatic components between the pre- and post-PTX periods in dialysis patients using the KDQOL-36 ${ }^{\mathrm{TM}}$.

\section{Patients and Methods}

\section{Data Sources}

We screened dialysis patients who had received PTX between July 1, 2016 and June 30, 2021 at the Dalian University Affiliated Xinhua Hospital, China. The inclusion criteria were (1) adult dialysis patients ( $\geq 20$ years); (2) attended regular outpatient hemodialysis (HD) sessions thrice a week and peritoneal dialysis (PD). The exclusion criteria were as follows: (1) patients who had initiated regular HD or PD after July 1, 2016; (2) patients who were older than 90 years; (3) patients transferred to other medical facilities; and (4) patients whose information was incomplete and/or those who were lost to follow-up during the study period. A total of 420 patients were screened for inclusion in the study. Among them, we defined individuals who underwent PTX, and 212 patients who underwent PTX were included in the final study analysis (Figure 1).

The protocol of management hyperparathyroidism in dialysis patients was follow-up KDIGO chronic kidney diseasemineral bone disease (CKD-MBD) clinical practice guideline. ${ }^{20}$ The indications for parathyroidectomy were uncontrolled pruritus, generalized bone pain, resistance to medical treatment, and high intact PTH levels (>1000 pg/mL). The

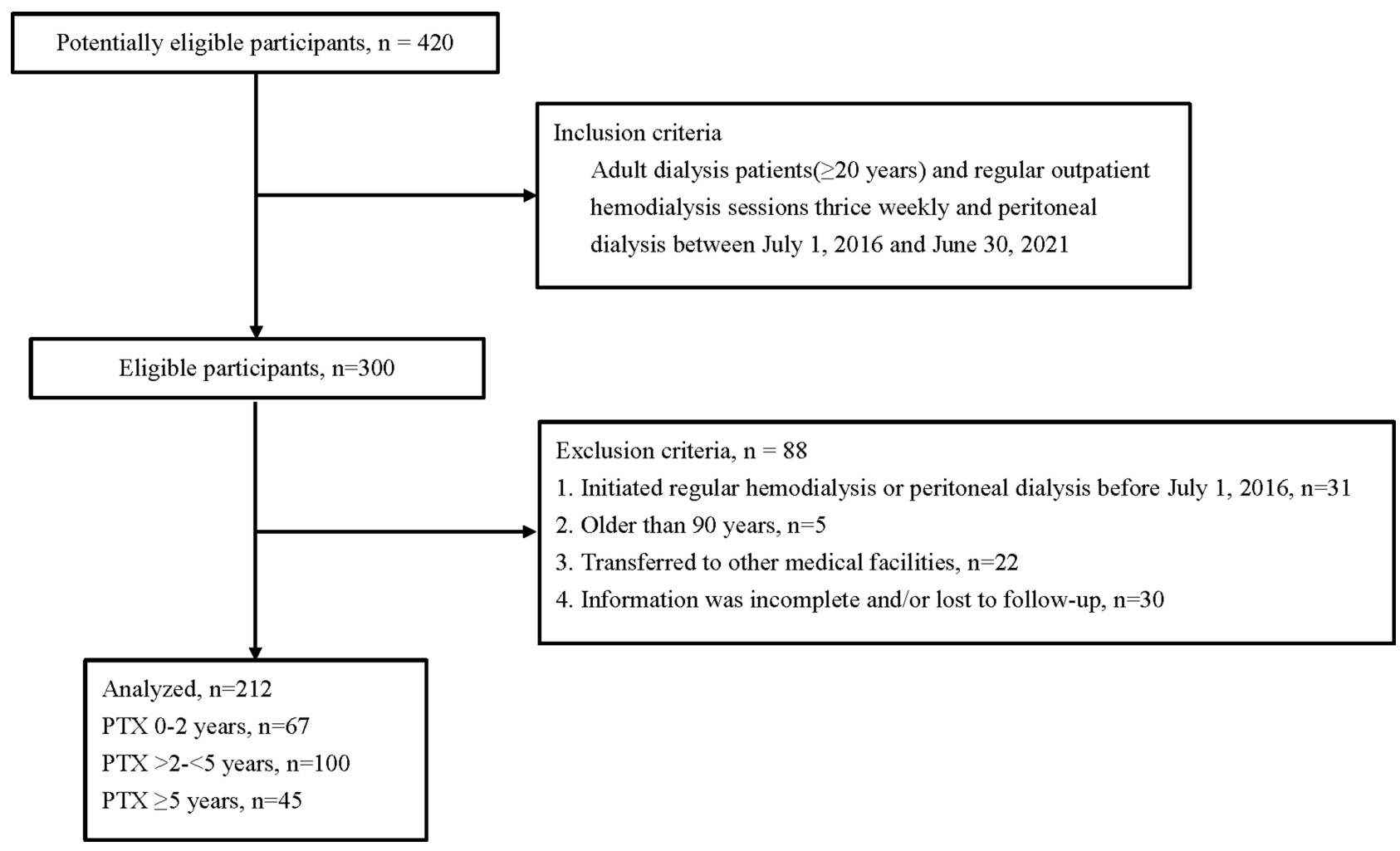

Figure I Participant deposition. 
parathyroidectomy procedure included a total parathyroidectomy and auto-transplantation of $140 \mathrm{mg}$ of hyperplastic tissue of the parathyroid gland into the subcutaneous forearm tissue.

\section{Laboratory Measurement}

Laboratory blood values were usually measured monthly, except for intact PTH levels, which were measured every 3 months, and urea clearance determined by kinetic modeling (Kt/V urea) (Daugirdas method), ${ }^{21}$ which was measured every 6 months. Baseline parameters were defined as the last blood test pre-PTX and the mean of the three consecutive monthly tests prior to censoring (July 1, 2021) for follow-up data. All blood samples were measured using commercial kits and an autoanalyzer (ADVIA1800, Siemens Healthcare Diagnostic Inc., NY, USA), and intact PTH levels were measured using chemiluminescence immunoassay (ADVIA Centaur 240, Siemens Healthcare Diagnostic Inc., NY, USA). Albumin levels were measured using the bromocresol green method. The indications for PTX were uncontrolled pruritus, generalized bone pain, resistance to medical treatment, and high intact PTH concentrations ( $>1000 \mathrm{pg} / \mathrm{mL})$. The PTX procedure included total PTX and autotransplantation of $140 \mathrm{mg}$ of hyperplastic tissue of the parathyroid gland into the subcutaneous forearm tissue.

\section{Survey Instrument for QoL}

The psychometric measurement used in this study was the KDQOL-36 $6^{\mathrm{TM}}$ version 1.3. The KDQOL-36 ${ }^{\mathrm{TM}}$ is available in English and translated into Mandarin Chinese by the KDQOL-SFTM group and RAND (The KDQOL Working Group. https://www.rand.org/health-care/surveys tools/kdqol.html). The KDQOL-36 ${ }^{\mathrm{TM}}$ comprises generic and disease-specific domains. The disease-specific domains comprise three scales: Symptoms and Problems of Kidney Disease (SPKD) (12 items), Burden of Kidney Disease (BKD) (4 items), and Effects of Kidney Disease (EKD) (8 items). The generic core is the 12-item Short Form Health Survey (SF-12). The results of the SF-12 instrument are summarized as the Physical Component Summary (PCS) score and Mental Component Summary (MCS) score. Scoring of the subscales was available from the free scoring guide (https://www.rand.org/health-care/surveys_tools/kdqol.html). PCS and MCS scores were determined using a T-score metric. SPKD, BKD, and EKD scores ranged from 0 to 100, with higher scores indicating better QoL. The pre-PTX measurement was performed 1 month prior to the PTX. The cross-sectional postPTX measurement was initiated from June 1, 2021 to June 31, 2021.

\section{Compliance with Ethical Standards}

Ethical approval: All procedures performed in studies involving human participants were in accordance with the ethical standards of the institutional and/or national research committee at which the studies were conducted [Institutional Review Board (IRB) approval number 1.0, 2021.8.11 in Dalian University Affiliated Xinhua Hospital, China] and with the 1964 Helsinki declaration and its later amendments or comparable ethical standards.

Informed consent: Informed consent was obtained from all individual participants included in the study.

\section{Statistical Analysis}

Baseline characteristics were calculated using descriptive statistics (mean \pm standard deviation) and percentages. Differences between the groups were estimated using the chi-square test and independent two-sample $t$-test. The correlation between the KDQOL-36 components and clinical variables was estimated using the Pearson correlation test. Statistical significance was set at $P<0.05$. All statistical analyses were conducted using the Statistical Package for Social Sciences (version 25.0).

\section{Results}

\section{Characteristics of the Participants}

The cohort comprised 212 patients, with a mean age of 46.4 years, and the male:female ratio was 96:116. Among them, 191 patients received HD and 21 patients received PD. Regarding comorbidities, 69.8\% ( $\mathrm{n}=148)$ had hypertension, $26.4 \%(n=56)$ had diabetes, and $10.8 \%(n=23)$ had coronary artery disease (Table 1$)$. 
Table I Baseline Characteristics $(\mathrm{N}=2 / 2)$

\begin{tabular}{|l|l|}
\hline Variables & n (\%) \\
\hline Sex (M/F) & $96 / 116$ \\
Age (year) & $46.4 \pm 11.3$ \\
Dialysis vintage (year) & $10.0 \pm 4.0$ \\
Hemodialysis & $191(90.0)$ \\
Peritoneal dialysis & $21(10.0)$ \\
Diabetes & $56(26.4)$ \\
Hypertension & $148(69.8)$ \\
Coronary cardiac disease & $23(10.8)$ \\
Chronic hepatitis, B/C & $11 / 15(12.3)$ \\
\hline
\end{tabular}

\section{Comparison of the Laboratory Parameters in the Pre- and Post-PTX Periods}

Nutritional markers, albumin, significantly increased post PTX than pre PTX. CKD-mineral bone disease parameters, including calcium, phosphate, alkaline phosphatase, and intact PTH, significantly decreased post PTX than pre PTX (Table 2).

\section{Comparison of KDQOL-36 Domain Scale Scores in the Pre- and Post-PTX Periods}

In the analysis of all patients, the KDQOL-36 domain scale scores (PCS, MCS, BKD, SPKD, and EKD) significantly increased in the post-PTX period compared with the pre-PTX period (Table 3). We further stratified the patients into three groups based on the duration of PTX: $0-2$ years, $>2-<5$ years, and $\geq 5$ years. All KDQOL- 36 domain scale scores increased with the duration of PTX treatment (Table 4).

Table 2 Distribution of Laboratory Parameters Pre- and Post-Parathyroidectomy (PTX) Periods ( $\mathrm{n}=2 \mathrm{I} 2$ )

\begin{tabular}{|l|l|l|l|}
\hline Parameters & Pre-PTX & Post-PTX & P \\
\hline Hemoglobin (g/L) & $116.39 \pm 19.49$ & $112.64 \pm 15.41$ & 0.027 \\
Albumin (g/L) & $41.55 \pm 3.23$ & $44.62 \pm 3.30$ & $<0.00 \mathrm{I}$ \\
Urea nitrogen (umol/L) & $26.57 \pm 6.22$ & $19.84 \pm 5.93$ & $<0.00 \mathrm{I}$ \\
Creatinine (umol/L) & $924.17 \pm 362.39$ & $819.21 \pm 166.42$ & $<0.00 \mathrm{I}$ \\
Uric acid (umol/L) & $463.35 \pm 70.27$ & $383.73 \pm 98.92$ & $<0.00 \mathrm{I}$ \\
Calcium (mmol/L) & $2.64 \pm 1.42$ & $2.10 \pm 0.40$ & $<0.00 \mathrm{I}$ \\
Phosphate (mmol/L) & $2.13 \pm 0.54$ & $1.82 \pm 0.50$ & $<0.00 \mathrm{I}$ \\
Potassium (mmol/L) & $4.99 \pm 2.53$ & $4.95 \pm 0.77$ & 0.082 \\
ALP (u/L)* & $322(135-78 \mathrm{I})$ & $268(108-422)$ & $<0.00 \mathrm{I}$ \\
iPTH (pg/mL)* & $1812(1171.25-2640.73)$ & $13.9(5.00-119.70)$ & $<0.00 \mathrm{I}$ \\
Kt/V & $1.19 \pm 0.13$ & $1.25 \pm 0.10$ & $<0.00 \mathrm{I}$ \\
\hline
\end{tabular}

Notes: Data are expressed as mean $\pm \mathrm{SD}$, *expressed as median (interquartile range).

Abbreviations: iPTH, intact parathyroid hormone; ALP, alkaline phosphatase.

Table 3 KDQOL-36 Domain Scale Scores, Pre-and Post-PTX Periods

\begin{tabular}{|l|l|l|l|}
\hline & Pre-PTX & Post-PTX & P \\
\hline PCS & $34.28 \pm 4.84$ & $46.01 \pm 6.19$ & $<0.001$ \\
MCS & $47.08 \pm 6.75$ & $51.99 \pm 5.22$ & $<0.001$ \\
BKD & $11.54 \pm 10.41$ & $21.72 \pm 13.84$ & $<0.001$ \\
SPKD & $69.17 \pm 14.92$ & $87.27 \pm 12.5$ & $<0.001$ \\
EKD & $70.43 \pm 12.64$ & $76.01 \pm 13.37$ & $<0.001$ \\
\hline
\end{tabular}

Abbreviations: BKD, burden of kidney disease; EKD, effects of kidney disease; MCS, mental component summary; PCS, physical component summary; PTH, parathyroid hormone; SPKD, symptoms and problems of kidney disease. 
Table 4 KDQOL-36 Domain Scale Scores in Stratified PTX Duration

\begin{tabular}{|c|c|c|c|c|c|c|c|c|c|c|}
\hline & \multicolumn{2}{|c|}{ PCS } & \multicolumn{2}{|c|}{ MCS } & \multicolumn{2}{|c|}{ BKD } & \multicolumn{2}{|c|}{ SPKD } & \multicolumn{2}{|c|}{ EKD } \\
\hline & Pre-PTX & Post-PTX & Pre-PTX & Post-PTX & Pre-PTX & Post-PTX & Pre-PTX & Post-PTX & Pre-PTX & Post-PTX \\
\hline $\begin{array}{l}\text { PTX 0-2 } \\
\text { years, } n=67\end{array}$ & $35.02 \pm 5.6$ & $44.78 \pm 5.66 * *$ & $45.66 \pm 7.74$ & $51.62 \pm 5.34^{* *}$ & $10.94 \pm \mid 1.51$ & $21.88 \pm 13.9 * *$ & $73.26 \pm|2.8|$ & $85.94 \pm 8.89 * *$ & $73.96 \pm 12.59$ & $79.08 \pm 13.89 * *$ \\
\hline $\begin{array}{l}\mathrm{PTX}>2-<5 \\
\text { years, } n=100\end{array}$ & $34.35 \pm 4.61$ & $46.95 \pm 5.92 * *$ & $46.77 \pm 7.23$ & $51.71 \pm 5.16^{* *}$ & $11.63 \pm 9.91$ & $20.14 \pm 13.03 * *$ & $69.68 \pm 13.36$ & $83.92 \pm 9.12^{* * *}$ & $70.66 \pm 13.11$ & $77.08 \pm 13.03^{* *}$ \\
\hline $\begin{array}{l}\text { PTX } \geq 5 \\
\text { years, } n=45\end{array}$ & $33.55 \pm 4.3$ & $44.34 \pm 6.58 * *$ & $49.43 \pm 5.31$ & $54.17 \pm 3.39 * *$ & $12.04 \pm 9.79$ & $23.15 \pm 14.59 *$ & $64.58 \pm 10.93$ & $81.94 \pm 7.6 * *$ & $66.67 \pm 12.23$ & $71.88 \pm 13.2 *$ \\
\hline
\end{tabular}

Note: $* \mathrm{P}<0.01, * * \mathrm{P}<0.001$.

\section{SPKD Scores in the Pre- and-Post-PTX Periods}

All individual patient-reported symptom scores, such as anorexia, dizziness, shortness of breath, pruritus, dry skin, cramps, chest pain, and bone pain, decreased in the post-PTX period compared with those in the pre-PTX period. The markedly decreased scores in the post-PTX period were found in the case of pruritus, dry skin, cramps, and bone pain (Figure 2).

\section{Pearson's Correlation Analysis on the Study Parameters}

PTX duration showed a significant negative correlation between PCS subscale scores $(P=0.001)$ and a positive correlation between EKD subscale scores $(P=0.004)$. Moreover, the SPKD subscale scores showed a significant negative correlation between age $(P=0.004)$ and a positive correlation between calcium $(P=0.021)$ (Table 5).

\section{Discussion}

It is well recognized that secondary HPT confers adverse health outcomes in CKD. ${ }^{22} \mathrm{PTX}$ is an option for CKD patients with medically refractory secondary HPT, illustrating favorable long-term outcomes in observational cohort studies. ${ }^{11,23}$ Considering the improvement in clinical symptoms caused by PTX, it is reasonable to assume QoL improvement after PTX in patients with CKD. Commonly, the QoL assessment tool in CKD comprise general health scales and kidney-

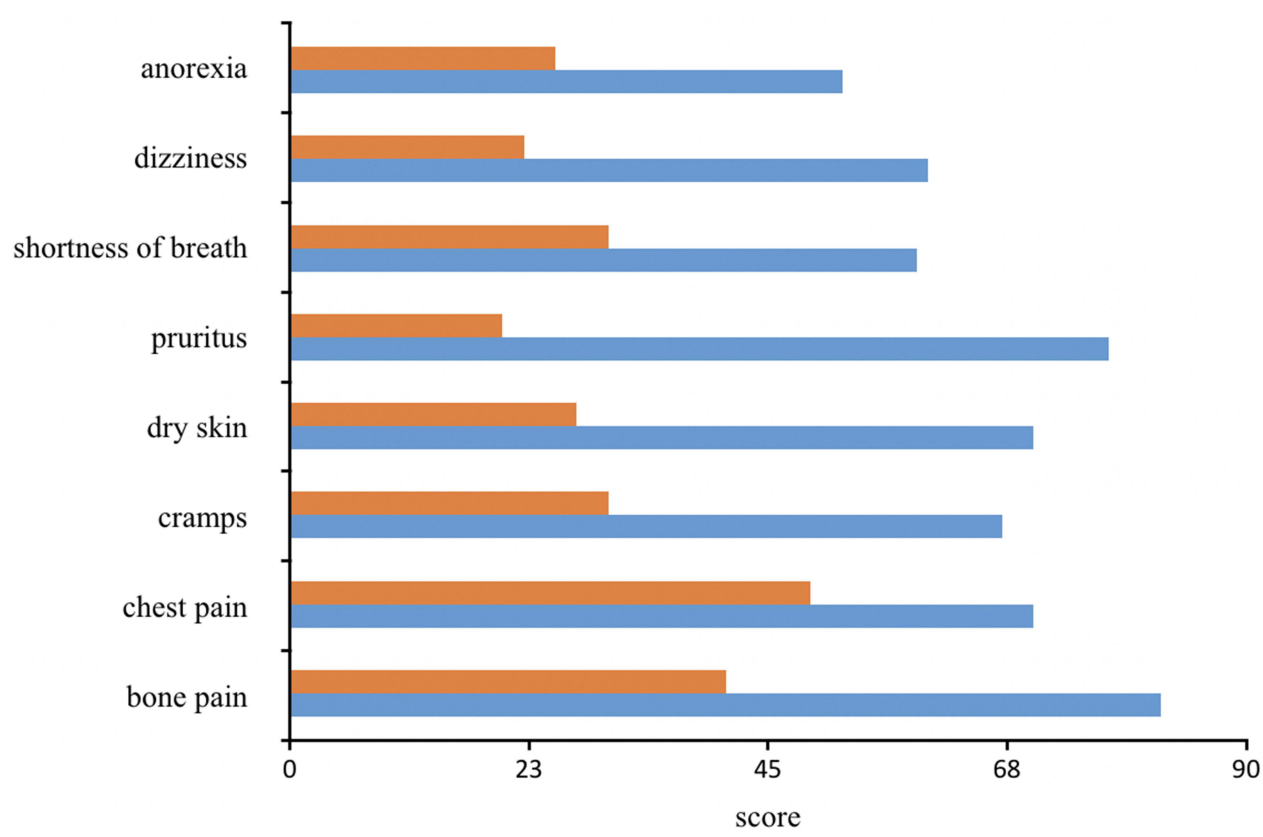

Figure 2 Distribution of Symptoms and Problems of Kidney Disease scale scores in the pre- and post-PTX periods. Data are expressed as mean values. $P<0.00 \mathrm{I}$.

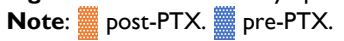


Table 5 Pearson's Correlation Analysis Between Studies Variables

\begin{tabular}{|c|c|c|c|c|c|c|c|c|c|c|}
\hline & \multicolumn{2}{|c|}{ PCS } & \multicolumn{2}{|c|}{ MCS } & \multicolumn{2}{|c|}{ BKD } & \multicolumn{2}{|c|}{ SPKD } & \multicolumn{2}{|c|}{ EKD } \\
\hline & $\mathbf{r}$ & $\mathbf{P}$ & $r$ & $\mathbf{P}$ & $\mathbf{r}$ & $\mathbf{P}$ & $\mathbf{r}$ & $\mathbf{P}$ & $\mathbf{r}$ & $\mathbf{P}$ \\
\hline Age & 0.041 & 0.388 & -0.043 & 0.365 & 0.022 & 0.639 & -0.137 & 0.004 & -0.056 & 0.242 \\
\hline Dialysis vintage & 0.025 & 0.617 & 0.034 & 0.483 & 0 & 0.998 & -0.08 & 0.103 & -0.058 & 0.24 \\
\hline Hemoglobin & -0.032 & 0.503 & 0.021 & 0.653 & -0.006 & 0.898 & 0.021 & 0.666 & 0.085 & 0.077 \\
\hline Albumin & 0.044 & 0.358 & 0.067 & 0.161 & 0.032 & 0.504 & 0.02 & 0.674 & -0.004 & 0.928 \\
\hline Calcium & -0.052 & 0.276 & 0.03 & 0.528 & -0.001 & 0.977 & 0.109 & 0.021 & 0.088 & 0.063 \\
\hline Phosphate & -0.01 & 0.838 & -0.027 & 0.561 & 0.01 & 0.828 & -0.056 & 0.237 & -0.002 & 0.958 \\
\hline Duration PTX & -0.180 & 0.001 & -0.027 & 0.6 & 0.085 & 0.079 & -0.07 & 0.17 & 0.146 & 0.004 \\
\hline iPTH & -0.109 & 0.021 & -0.01 & 0.83 & -0.01 & 0.826 & -0.041 & 0.383 & 0.021 & 0.654 \\
\hline
\end{tabular}

target scales. Given the nature of patient-reported forms in the tool, individuals vary in their ability to respond to the questionnaires, contributing to further intraindividual variability. Hence, the literature reports varied results. Considering the durable effects of PTX on QoL in dialysis patients, we conducted this study. Our study highlights that PTX could improve QoL components in dialysis patients.

In the present study, we used KDQOL-36 ${ }^{\mathrm{TM}}$ to measure QoL in dialysis patients during the pre- and post-PTX periods. The Chinese version of the KDQOL-36 ${ }^{\mathrm{TM}}$ has been demonstrated to be valid and reliable in dialysis populations who speak Mandarin. ${ }^{24-26}$ The drawback of the Chinese version of KDQOL-36 is the lack of a T-score metric in PCS and MCS subscale scores. Alternatively, we used reference values of the T-score metric in the Asian dialysis population reported in the US study. ${ }^{19,27}$ Our results showed that PCS and MCS subscale scores in our cohort exceeded the mean values reported in the Asian-US population. This implies that PTX may have a role in relieving physical and mental complex symptoms in the patients included in our study. Regarding symptom relief, pruritus, dry skin, cramps, and bone pain were especially marked in the post-PTX period. PTH is a uremic toxin that contributes to complex calciumphosphate balance and multiorgan involvement. ${ }^{28}$ Thus, decreased PTH concentrations could potentially explain the improvement in PTH-related clinical symptoms. Symptom burden and high level of disability are common in dialysis patients, and the executive summary of the KDIGO Controversies Conference on Support Care in CKD emphasizes difficulties in developing a treatment regimen for symptom management in $\mathrm{CKD} .{ }^{29}$ Although our results need to be further validated by screening tools, we expect PTX to be a potential management regimen for medically refractory secondary HPT in dialysis patients.

Previous studies investigated the effect of PTX on QoL between 6 and 12 months. ${ }^{4,14,15,17}$ The reason is to minimize interventional bias and patient expectations of the PTX effect. Briefly, these studies demonstrated that PTX had a beneficial effect on clinical outcomes and improvement in QoL scales. In the present study, we found that improvement in QoL scale scores showed a consistent pattern in the stratified PTX duration. On further analysis, our cohort showed increased serum albumin concentrations after PTX. Given the myriad of symptoms related to PTH, we suppose that improved QoL scales, particularly in the physical health domain, may be linked to beneficial effects on clinical parameters after PTX, such as nutrition, and calcium-phosphate homeostasis. Indeed, we cannot refute the contribution of other clinical factors in QoL improvement in our post-PTX cohort, such as dialysis adequacy, category of hemodialyzer and clearance of various unmeasured uremic toxins. A further comprehensive and informative evaluation in QoL improvement in post-PTX dialysis patients warrants to be continued. However, PTX duration showed a significant negative correlation between PCS subscale scores and a positive correlation between EKD subscale scores. These results may be biased owing to the small sample size. Nonetheless, a long-term effect on QoL after PTX is certainly affected by various uremic complications and the quality of health care providers.

Although the present study provided promising evidence of the PTX effect on QoL in dialysis patients, there were some limitations to the methods used. First, the questionnaires for QoL evaluation were conducted at various time points post-PTX in an individual patient. The results may confer a heterogeneous distribution of the KDQOL-36 ${ }^{\mathrm{TM}}$ subscale scores. Although we have tried to analyze the KDQOL-36 $6^{\mathrm{TM}}$ subscale scores using stratification of different years post- 
PTX in the entire cohort, we admit that this stratified method cannot intrinsically realize the longitudinal effects of PTX on QoL in individual dialysis patients. Second, the PCS and MCS were scored on a T-score metric using a free scoring guide on a website (https://www.rand.org/health-care/surveys tools/kdqol.html). To the best of our knowledge, there is still a lack of T-score metrics for PCS and MCS in Chinese dialysis patients. Therefore, we used reference values of the T-score metric in the Asian dialysis population reported in a United States study. ${ }^{19}$ Hence, the validity and reliability of PCS and MCS measurements in Chinese dialysis patients need to be further investigated. We admit that this approach may result in bias in subscale scores for PCS and MCS. This approach also makes it difficult to compare the effects of PTX on PCS and MCS in various dialysis populations worldwide. However, the present study investigated a cohort dialysis population in a single dialysis center within a limited period. Hence, the trajectory of PCS and MCS scale scores was not easily influenced by a non-generalized health care protocol and various environmental habits in the dialysis population. We believe that trend changes in the components of KDQOL-36 $6^{\mathrm{TM}}$ between pre- and post-PTX periods are still reliable in the present study. Third, this study used a patient-reported QoL questionnaire survey. It is reasonable to assume that several patient perspectives could influence responses to questionnaires. Consequently, interpretation bias is unavoidable. Finally, our study included a relatively small number of dialysis patients in a hospital-facilitated dialysis center. The results could be influenced by patient characteristics, quality of health care providers, and heterogeneous habitual factors. Therefore, a detailed conclusion of the effects of PTX on QoL in dialysis patients may not be extrapolated to other dialysis populations.

\section{Conclusion}

PTX could improve QoL in dialysis patients with medically refractory secondary HPT. The long-term effects merit to be studied using a large sample.

\section{Acknowledgments}

The authors would like to appreciate Professor Zhang Ling, associated with the China-Japan Friendship Hospital, Beijing, China, for her academic recommendation for the content of the manuscript.

\section{Disclosure}

All the authors have declared no competing interest in this work.

\section{References}

1. Kidney Disease: Improving Global Outcomes (KDIGO) CKD-MBD Update Work Group. KDIGO 2017 Clinical Practice Guideline Update for the Diagnosis, Evaluation, Prevention, and Treatment of Chronic Kidney Disease-Mineral and Bone Disorder (CKD-MBD). Kidney Int Suppl. 2017;7:1-59. doi:10.1016/j.kisu.2017.04.001

2. Ketteler M, Block GA, Evenepoel P, et al. Executive summary of the 2017 KDIGO Chronic Kidney Disease-Mineral and Bone Disorder (CKDMBD) guideline update: what's changed and why it matters. Kidney Int. 2017;92:26-36. doi:10.1016/j.kint.2017.04.006

3. Lee CT, Chou FF, Chang HW, et al. Effects of parathyroidectomy on iron homeostasis and erythropoiesis in hemodialysis patients with severe hyperparathyroidism. Blood Purif. 2003;21:369-375. doi:10.1159/000073438

4. Chow KM, Szeto CC, Kum LC, et al. Improved health-related quality of life and left ventricular hypertrophy among dialysis patients treated with parathyroidectomy. J Nephrol. 2003;16:878-885.

5. Chou FF, Chen JB, Hsieh KC, Liou CW. Cognitive changes after parathyroidectomy in patients with secondary hyperparathyroidism. Surgery. 2008;143:526-532. doi:10.1016/j.surg.2007.11.019

6. Chou FF, Lee CH, Chen JB, Huang SC, Lee CT. Sleep disturbances before and after parathyroidectomy for secondary hyperparathyroidism. Surgery. 2005;137:426-430. doi:10.1016/j.surg.2004.12.010

7. Raggi P, Boulay A, Chasan-Taber S, et al. Cardiac calcification in adult hemodialysis patients. A link between end-stage renal disease and cardiovascular disease? J Am Coll Cardiol. 2002;39:695-701. doi:10.1016/S0735-1097(01)01781-8

8. Ursem SR, Heijboer AC, D'Haese PC, et al. Non-oxidized parathyroid hormone (PTH) measured by current method is not superior to total PTH in assessing bone turnover in chronic kidney disease. Kidney Int. 2021;99(5):1173-1178. doi:10.1016/j.kint.2020.12.024

9. Ganesh SK, Stack AG, Levin NW, Hulbert-Shearon T, Port FK. Association of elevated serum PO(4), Ca x PO(4) product, and parathyroid hormone with cardiac mortality risk in chronic hemodialysis patients. $J$ Am Soc Nephrol. 2001;12:2131-2138. doi:10.1681/ASN.V12102131

10. Block GA, Hulbert-Shearon TE, Levin NW, Port FK. Association of serum phosphorus and calcium x phosphate product with mortality risk in chronic hemodialysis patients: a national study. Am J Kidney Dis. 1998;31:607-617. doi:10.1053/ajkd.1998.v31.pm9531176

11. Chen JB, Chou FF, Yang CH, Hua MS. Association between clinical variables and mortality after parathyroidectomy in maintenance hemodialysis patients. Am J Surg. 2017;213:140-145. doi:10.1016/j.amjsurg.2016.03.012 
12. Tentori F, Wang M, Bieber BA, et al. Recent changes in therapeutic approaches and association with outcomes among patients with secondary hyperparathyroidism on chronic hemodialysis: the DOPPS study. Clin J Am Soc Nephrol. 2015;10:98-109. doi:10.2215/CJN.12941213

13. Weisbord SD, Carmody SS, Bruns FJ, et al. Symptom burden, quality of life, advance care planning and the potential value of palliative care in severely ill haemodialysis patients. Nephrol Dial Transplant. 2003;18:1345-1352. doi:10.1093/ndt/gfg105

14. Cheng SP, Lee JJ, Liu TP, et al. Parathyroidectomy improves symptomatology and quality of life in patients with secondary hyperparathyroidism. Surgery. 2014;155:320-328. doi:10.1016/j.surg.2013.08.013

15. Bratucu MN, Garofil ND, Radu PA, et al. Measurement of quality of life after total parathyroidectomy in patients with secondary hyperparathyroidism and end stage renal disease. Chirurgia (Bucur). 2015;110:511-517.

16. van der Plas WY, Dulfer RR, Engelsman AF, et al. Effect of parathyroidectomy and cinacalcet on quality of life in patients with end-stage renal disease-related hyperparathyroidism: a systematic review. Nephrol Dial Transplant. 2017;32:1902-1908. doi:10.1093/ndt/gfx044

17. El-Kholey M, Ibrahim GES, Elshahat OI, El-Kannishy G. Impact of subtotal parathyroidectomy on clinical parameters and quality of life in hemodialysis patients with secondary hyperparathyroidism. Endocrinol Metab (Seoul). 2019;34:367-373. doi:10.3803/EnM.2019.34.4.367

18. Filho WA, van der Plas WY, Brescia MDG, et al. Quality of life after surgery in secondary hyperparathyroidism, comparing subtotal parathyroidectomy with total parathyroidectomy with immediate parathyroid autograft: prospective randomized trial. Surgery. 2018;164:978-985. doi:10.1016/j.surg.2018.06.032

19. Peipert JD, Nair D, Klicko K, Schatell DR, Hays RD. Kidney Disease Quality of Life 36-item short form survey (KDQOL-36) normative values for the United States dialysis population and new single summary score. J Am Soc Nephrol. 2019;30:654-663. doi:10.1681/ASN.2018100994

20. Kidney Disease: Improving Global Outcomes (KDIGO) CKD-MBD Work Group. KDIGO clinical practice guideline for the diagnosis, evaluation, prevention, and treatment of Chronic Kidney Disease-Mineral and Bone Disorder (CKD-MBD). Kidney Int Suppl. 2009;113:S1-130.

21. Daugirdas JT. The post: pre-dialysis plasma urea nitrogen ratio to estimate K.t/V and NPCR: mathematical modeling. Int J Artif Organs. 1989;12:411-419.

22. Xu Y, Evans M, Soro M, Barany P, Carrero JJ. Secondary hyperparathyroidism and adverse health outcomes in adults with chronic kidney disease. Clin Kidney J. 2021;14:2213-2220. doi:10.1093/ckj/sfab006

23. Lau WL, Obi Y, Kalantar-Zadeh K. Parathyroidectomy in the management of secondary hyperparathyroidism. Clin J Am Soc Nephrol. 2018;13:952-961. doi:10.2215/CJN.10390917

24. Joshi VD, Mooppil N, Lim JF. Validation of the kidney disease quality of life-short form: a cross-sectional study of a dialysis-targeted health measure in Singapore. BMC Nephrol. 2010;11:36. doi:10.1186/1471-2369-11-36

25. Chow SK, Tam BM. Is the kidney disease quality of life-36 (KDQOL-36) a valid instrument for Chinese dialysis patients? BMC Nephrol. 2014;15:199. doi:10.1186/1471-2369-15-199

26. Lam CL, Tse EY, Gandek B. Is the standard SF-12 health survey valid and equivalent for a Chinese population? Qual Life Res. 2005;14:539-547. doi:10.1007/s11136-004-0704-3

27. Hays RD, Peipert JD, Kallich JD. Problems with analyses and interpretation of data in "use of the KDQOL-36 $6^{\mathrm{TM}}$ for assessment of health-related quality of life among dialysis patients in the United States". BMC Nephrol. 2019;20:447. doi:10.1186/s12882-019-1609-2

28. Komaba H, Kakuta T, Fukagawa M. Management of secondary hyperparathyroidism: how and why? Clin Exp Nephrol. 2017;21:37-45. doi:10.1007/s10157-016-1369-2

29. Davison SN, Levin A, Moss AH, et al. Executive summary of the KDIGO Controversies Conference on Supportive Care in Chronic Kidney Disease: developing a roadmap to improving quality care. Kidney Int. 2015;88:447-459. doi:10.1038/ki.2015.110

International Journal of General Medicine

Dovepress

\section{Publish your work in this journal}

The International Journal of General Medicine is an international, peer-reviewed open-access journal that focuses on general and internal medicine, pathogenesis, epidemiology, diagnosis, monitoring and treatment protocols. The journal is characterized by the rapid reporting of reviews, original research and clinical studies across all disease areas. The manuscript management system is completely online and includes a very quick and fair peer-review system, which is all easy to use. Visit http://www.dovepress.com/testimonials.php to read real quotes from published authors.

Submit your manuscript here: https://www.dovepress.com/international-journal-of-general-medicine-journal 